\title{
Aggregate Models based on Improved Group Methods for Simulation and Optimization of Distillation Systems
}

\author{
Ravindra S. Kamath ${ }^{1}$, Ignacio E. Grossmann, ${ }^{1,}$, and Lorenz T. Biegler ${ }^{1}$ \\ ${ }^{1}$ Chemical Engineering Department, Carnegie Mellon University, Pittsburgh PA 15213, USA
}

June 22, 2009

\begin{abstract}
This note deals with aggregate models for complex distillation systems in largescale flowsheets. Group methods were originally devised for simple absorber and stripper calculations with no major extensions for handling distillation. In this work, group methods are systematically analyzed and further improved by modifying some of the previously proposed approximations. As a result, the improved group method exhibits accurate predictions and this is demonstrated using simulation and optimization case studies for a variety of chemical systems and operating conditions. It is observed that the prediction of output variables is in close agreement with that of the rigorous equilibrium stage model. In case of optimization problems, the optimal number of trays and feed locations differ by only one or two trays. The aggregate model can be applied in a sequence of steps in order to improve the reliability and robustness of the solution procedure. A rounding heuristic is also proposed which can provide near-optimal solutions with a significant reduction in computational time.
\end{abstract}

\footnotetext{
*To whom correspondence should be addressed. Email: grossmann@cmu.edu
} 


\section{Introduction}

Countercurrent gas liquid operations are an important part of many chemical engineering applications. The most common and frequently occurring unit operation that involves countercurrent flow of vapor and liquid is distillation. With advances in algorithms and computational technology, the recent trend is to use rigorous tray-by-tray models for representing distillation columns. As an example, synthesis problems that determine the optimal number of trays and feed locations for a distillation column have been addressed using rigorous models (Viswanathan and Grossmann, 1990, 1993a,b; Yeomans and Grossmann, 2000; Lang and Biegler, 2002; Barttfeld et al., 2003). However, such optimization problems have usually been solved only for small flowsheets having only one or two columns. Use of such models in the optimization of more complex flowsheets having many distillation columns and other process equipments is challenging because of issues like nonlinearity, combinatorial complexity and convergence. Rigorous models should always be preferred because they are capable of representing the process behavior more accurately. However, if the resulting problem becomes too complex or intractable, a better approach would be to use aggregate models, particularly if their performance closely matches that of rigorous models.

Several types of aggregate models for distillation have been proposed in the literature. Some of them are based on shortcut/design (Kremser, 1930; Fenske, 1932; Underwood, 1948; Gilliland, 1940), heat/mass transfer (Bagajewicz and Manousiouthakis, 1992; Papalexandri and Pistikopoulos, 1996) and pinch/design (Caballero and Grossmann, 1999). However, most of these models cannot be directly used in process simulation and optimization mostly because of two issues. First, their accuracy is not as good as rigorous models like RADFRAC in Aspen Plus (AspenTech, 2006). Second, they are not suitable for generating complex column configurations or optimizing structural parameters like number of trays and feed locations. Group methods (Henley and Seader, 1981), which are based on aggregating a section of stages of the column have some potential but they have not been thoroughly investigated since their development in 1950s.

Group methods basically use approximate calculations to relate the outlet stream properties to the inlet stream specifications and number of equilibrium trays. These approximation procedures are called group methods because they provide only an overall treatment of the stages in the cascade without considering detailed changes in the temperature and composition of individual stages. However, they are much easier to solve because of fewer variables and constraints. They can be used to represent cascade of trays in many 

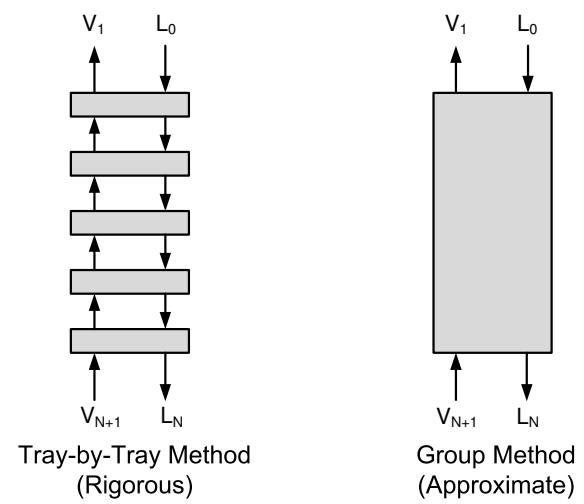

Figure 1: Rigorous and approximate group method for a counter-current cascade of trays

countercurrent operations like absorption, stripping, distillation, extraction, leaching etc.

In this paper, we investigate the potential of group methods for process simulation and optimization and propose further improvements specifically for modeling distillation columns. An equation-oriented analysis of group methods is presented along with various kinds of approximations used in previous work. As a next step, some of these approximations are replaced by more realistic constraints based on physical insights. As a result, the improved group method shows more accurate predictions and this is demonstrated using simulation and optimization case studies. It is observed that most of the output variables have less than $5 \%$ error, on average as compared to rigorous values and the optimal number of trays and feed locations are missed by only one or two trays. A rounding heuristic is also proposed which provides reasonably good solutions and significantly reduces the computational time for solving mixed integer nonlinear programming (MINLP) problems.

\section{Analysis of group methods}

Group methods were originally devised for simple hand calculations that are performed in an iterative manner. To investigate the previously proposed approximations in group methods, it is essential to perform a degree of freedom analysis to understand which approximations were used and for what purpose. A counter-current cascade of trays and its corresponding representation by group method is shown in Figure 1. The specifications for the entering vapor $V_{N+1}$ and the entering liquid $L_{0}$ serve as fixed inputs to the model. The approximate model based on group method evaluates the outlet stream properties 
like pressure, temperature, flow and composition of exiting vapor $V_{1}$ and exiting liquid $L_{N}$. For the sake of argument, we consider adiabatic and isobaric operation of the cascade. Thus, the pressure variables corresponding to the two outlet streams can be eliminated. If $C$ denotes the set of components involved in the system, then the total number of variables in the model is $2(|C|+1)$ due to the two outlet streams without the pressure variables.

The fundamental equations for group methods are the component mole balances,

$$
V_{N+1, i} y_{N+1, i}+L_{0, i} x_{0, i}=V_{1, i} y_{1, i}+L_{N, i} x_{N, i} \quad i \in C
$$

and the energy balance,

$$
V_{N+1} H_{N+1}^{V}+L_{0} H_{0}^{L}=V_{1} H_{1}^{V}+L_{N} H_{N}^{L}
$$

which are written around the complete set of trays forming the cascade. Note that (1) provides $|C|$ equations while (2) provides just one equation. The performance equation of the cascade is given by,

$$
v_{1, i}=v_{N+1, i} \phi_{A, i}+l_{0, i}\left(1-\phi_{S, i}\right) \quad i \in C
$$

Equation (3) was originally derived by Kremser (1930) for the design of absorbers where it was written only for the key component. Here, it is written for all the components and hence it results in $|C|$ equations. A quick analysis up to this point shows that there is one degree of freedom. The $\phi_{A, i}$ and $\phi_{S, i}$ in (3) denote the recovery factors for absorption and stripping and are given by,

$$
\phi_{A, i}=\frac{A_{e, i}-1}{A_{e, i}^{N+1}-1} ; \quad \phi_{S, i}=\frac{S_{e, i}-1}{S_{e, i}^{N+1}-1}, \quad i \in C
$$

Here, $A_{e, i}$ and $S_{e, i}$ stand for effective absorption and stripping factors and they represent average values of absorption and stripping factors for all trays contained in the cascade. The averaging scheme proposed by Edmister (1943),

$$
\begin{aligned}
A_{e, i} & =\left[A_{N, i}\left(A_{1, i}+1\right)+0.25\right]^{0.5}-0.5 \\
S_{e, i} & =\left[S_{1, i}\left(S_{N, i}+1\right)+0.25\right]^{0.5}-0.5
\end{aligned}
$$

uses absorption and stripping factors at the top $\left(A_{1, i}\right.$ and $\left.S_{1, i}\right)$ and bottom $\left(A_{N, i}\right.$ and $\left.S_{N, i}\right)$ 
of the cascade. These factors, are in turn calculated using the following expressions,

$$
\begin{aligned}
& A_{1, i}=\frac{L_{1}}{K_{1, i} V_{1}} ; \quad A_{N, i}=\frac{L_{N}}{K_{N, i} V_{N}} \\
& S_{1, i}=1 / A_{1, i} ; \quad S_{N, i}=1 / A_{N, i}, \quad i \in C
\end{aligned}
$$

Note that each of the equations from (4) to (6) will be used to evaluate the variable on the left hand side of these equations. Consequently, the degree of freedom of unity up till the point before equation (4) still remains unsatisfied. Furthermore, equation (6) introduces two new variables $L_{1}$ and $V_{N}$ that denote the liquid and vapor flow from the first and the last tray respectively. These variables were not defined previously in the model and hence two additional equations are needed to account for these variables. This, along with the previously unsatisfied degree of freedom of unity leads to three degrees of freedom. As will be seen in the next section, previous work on group methods accounted for these three degrees of freedoms using different kinds of approximations.

\section{Approximations used for group methods}

Kremser (1930) proposed the following three approximations:

$$
\begin{aligned}
& L_{1} \approx L_{0} \\
& V_{N} \approx V_{N+1} \\
& T_{N} \approx \frac{T_{0}+T_{N+1}}{2}
\end{aligned}
$$

Kremser (1930) also replaces the energy balance (2) by

$$
T_{1} \approx \frac{T_{0}+T_{N+1}}{2}
$$

Equation (7) suggests that the flow of entering liquid does not change much after passing through first tray. A similar approximation is made in (8) for entering vapor flow as it passes through the last stage. Equations (9) and (10) suggest that Kremser (1930) used identical approximations for temperatures of the vapor and liquid streams exiting the cascade and they are both considered to be equal to the arithmetic mean of the temperature of entering vapor and liquid streams. Note that Kremser (1930) originally made these approximations for recovery of gasoline components from natural gas where only a small fraction of the feed gas is absorbed. The next major improvement to group methods was 
proposed by Edmister (1957) for handling distillation systems. Unlike Kremser (1930), Edmister (1957) retains the energy balance (2) for the cascade. However, he uses a different set of approximation equations depending upon whether the cascade behaves as an absorber or a stripper. The three approximation equations corresponding to behavior as an absorber are

$$
\begin{aligned}
& V_{N} \approx V_{N+1}\left(\frac{V_{1}}{V_{N+1}}\right)^{1 / N} \\
& L_{1}=L_{0}+V_{2}-V_{1} \\
& \frac{T_{N}-T_{1}}{T_{N}-T_{0}} \approx \frac{V_{N+1}-V_{2}}{V_{N+1}-V_{1}}
\end{aligned}
$$

Equation (11) provides an approximate expression for $V_{N}$ and assumes that the vapor contraction per stage is the same percentage of the vapor flow to the stage in question. Equation (13) is a relation between temperature and vapor flowrates and assumes that the temperature change of the liquid is proportional to the volume of gas absorbed. Although equation (12) is a rigorous expression for $L_{1}$ based on mole balance, it contains a new variable $V_{2}$ not defined previously. $V_{2}$ is approximated by the following equation, which is analogous to (11):

$$
V_{2} \approx V_{1}\left(\frac{V_{N+1}}{V_{1}}\right)^{1 / N}
$$

The approximation equations for the stripper are similar to that of absorber except that most of the dependencies are based on molar flow of liquid instead of vapor. These equations are as follows

$$
\begin{aligned}
& L_{1} \approx L_{0}\left(\frac{L_{N}}{L_{0}}\right)^{1 / N} \\
& V_{N}=V_{N+1}+L_{N-1}-L_{N} \\
& \frac{T_{0}-T_{1}}{T_{0}-T_{N}} \approx \frac{L_{0}-L_{1}}{L_{0}-L_{N}} \\
& L_{N-1} \approx L_{N}\left(\frac{L_{0}}{L_{N}}\right)^{1 / N}
\end{aligned}
$$

Clearly, some physical insight about the separation system such as chemical components involved and the operating conditions is required in order to identify whether a cascade of trays behaves as an absorber or a stripper. Although identifying this behavior is trivial for conventional absorbers and strippers, it is not obvious when many such cascades are connected together as in multicomponent distillation involving multiple feeds and/or 
side-draws.

\section{Proposed modifications}

Our proposed modifications involve identifying three constraints to satisfy the degrees of freedom as described earlier. The first two of our proposed constraints are based on the underlying principles of distillation. We expect that since the outlet streams are coming out of first and last tray of the cascade, they should be under vapor liquid equilibrium conditions and an aggregate model, of whatever order of simplicity, should capture these phenomena just like mass and energy balances. Hence, for the outlet vapor, we impose a constraint that it should be at dew point conditions i.e.

$$
\sum_{i \in C} \frac{y_{1, i}}{K_{1, i}}=1
$$

Similarly, the outlet liquid is at bubble point conditions i.e.

$$
\sum_{i \in C} K_{N, i} x_{N, i}=1
$$

It is worth mentioning that equations (19) and (20) are implicit in equilibrium stage models, and thus they can be considered to be physically realistic constraints for group methods. If needed, these equations can be appropriately modified for non-equilibrium behavior. Only our third equation given by

$$
L_{1}-L_{N} \approx V_{1}-V_{N}
$$

is an approximation. Equation (21) is based on an approximation of mole balance with an assumption that the decrease in vapor at the bottom is approximately equal to the increase in liquid at the top and vice versa. Note that it is a linear constraint and unlike the approach by Edmister (1957), this single equation can handle both absorption and stripping effects. The model given by (1) - (6) along with (19) - (21) forms a general purpose group model for a cascade of trays in a distillation column. Aggregate models

for any complex columns configurations can be easily generated by connecting such group models to rigorous or aggregate process models of condenser, reboiler, feed or side-draw stages through input and output streams interconnections.

As will be shown in the next section, the use of our proposed modifications leads to an 
Table 1: Data for the simulation case study

\begin{tabular}{|c|c|c|c|}
\hline \multirow{5}{*}{$\begin{array}{l}\text { Column configuration } \\
\text { Total number of stages } \\
\text { Location of first feed } \\
\text { Location of second feed } \\
\text { Location of vapor side-draw }\end{array}$} & \multicolumn{3}{|c|}{ Feed characteristics } \\
\hline & 45 & - Main and $2^{\text {nd }}$ feed & \\
\hline & $32^{\text {nd }}$ stage & Molar flow rate $(\mathrm{kmol} / \mathrm{s})$ & 3.0 \\
\hline & $21^{\text {st }}$ stage & $\mathrm{N}_{2}$ fraction $(\mathrm{mol} \%)$ & 70.8155 \\
\hline & $9^{\text {th }}$ stage & $\begin{array}{l}\mathrm{O}_{2} \text { fraction }(\mathrm{mol} \%) \\
\mathrm{Ar} \text { fraction }(\mathrm{mol} \%)\end{array}$ & $\begin{array}{l}27.9439 \\
1.2407\end{array}$ \\
\hline Operating conditions & & Temperature (mol \%) & 96.7 \\
\hline Column pressure (bar) & 5 & - Top feed & \\
\hline Reboil ratio & 3.5 & $\begin{array}{l}\text { Molar flowrate }(\mathrm{kmol} / \mathrm{s}) \\
\mathrm{N}_{2} \text { fraction }(\mathrm{mol} \mathrm{\%})\end{array}$ & $\begin{array}{l}1.0 \\
99.6717\end{array}$ \\
\hline Side-draw specification & & $\mathrm{O}_{2}$ fraction $(\mathrm{mol} \%)$ & 0.2687 \\
\hline Molar flowrate $(\mathrm{kmol} / \mathrm{s})$ & 2.0 & $\begin{array}{l}\text { Ar fraction }(\mathrm{mol} \%) \\
\text { Temperature }(\mathrm{mol} \%)\end{array}$ & $\begin{array}{l}0.0586 \\
93.8\end{array}$ \\
\hline
\end{tabular}

improved group method with better predictive capabilities for distillation. In our opinion, it would be unfair to compare the improved group method with that of Kremser (1930) because that work was tailored for absorption under mild conditions. Comparison with the work of Edmister (1957) is more appropriate, but is not straightforward. This is because for complex columns having $n$ cascades $(n>2)$, Edmister (1957)'s methodology will result in $2^{n}$ different solutions depending on what kind of behavior (absorber or stripper) is specified for each of the existing cascades. Then, it is not clear which of these solutions (or the behavior of the cascades) is the most appropriate representation of the column behavior and suitable for comparison with our aggregate model or the rigorous model. This aspect is highlighted using a simulation example in the next section.

\section{Simulation case study using aggregate models}

The effectiveness of using the improved group method in aggregate models for distillation columns is first demonstrated using a simulation case study. A complex column is considered which closely represents the low pressure column in cryogenic air separation. This column has two conventional feeds with a cascade of trays in between, a vapor sidedraw with a cascade of trays above and below it, and a liquid feed at the top instead of a condenser. The specifications used for the simulation of this column are given in Table 1. An aggregate representation of this complex column is shown in Figure 2 where each of the four set of contiguous stages is represented by the improved group method. The process models for the reboiler, feed stages and the side-draw stage in this aggregate representation are rigorous just like in the equilibrium tray-by-tray model. A comparison of the simulation results using the aggregate model and the rigorous model (RADFRAC of Aspen Plus) is shown in Table 2. Also included in Table 2 is the corresponding result 


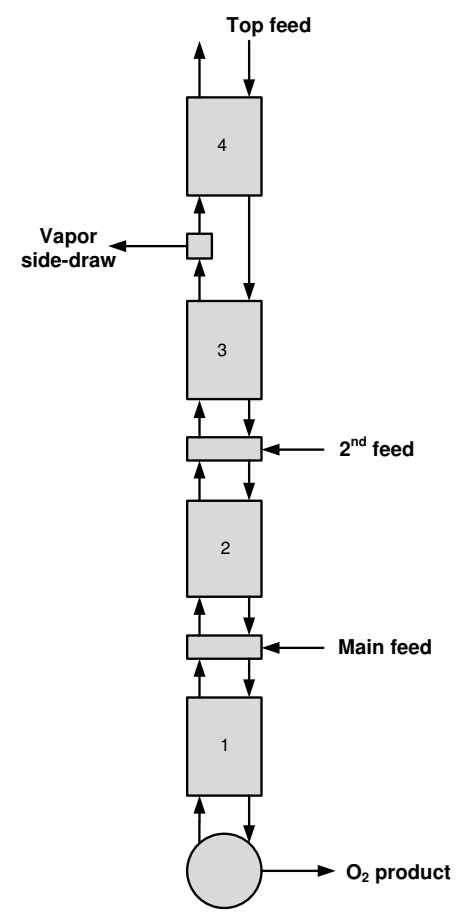

Figure 2: Aggregate representation of the complex column for the simulation case study

using the Edmister (1957) method. As discussed earlier, the methodology of Edmister (1957) requires selecting independently the behavior (absorption or stripping) for the four cascades thus resulting in sixteen possible solutions. The comparison in Table 2 uses the solution that most closely resembles the result of the rigorous model. As can be seen, most

Table 2: Results for simulation case study

\begin{tabular}{lccc}
\hline & $\begin{array}{c}\text { Rigorous Model } \\
\text { (Aspen Plus) }\end{array}$ & $\begin{array}{c}\text { Aggregate Model } \\
\text { (Improved Group Method) }\end{array}$ & $\begin{array}{c}\text { Aggregate Model } \\
\text { (Edmister, 1957) }\end{array}$ \\
\hline Top Product & & & \\
Flowrate (kmol/s) & 3.6557 & 3.6555 & 3.6355 \\
$\mathrm{~N}_{2}$ fraction & 0.9552 & 0.9553 & 0.9584 \\
$\mathrm{O}_{2}$ fraction & 0.0409 & 0.0411 & 0.0382 \\
Ar fraction & 0.0038 & 0.0036 & 0.0033 \\
Temperature (K) & 95.236 & 95.237 & 94.342 \\
Bottom Product & & & \\
Flowrate (kmol/s) & 1.3442 & 1.3445 & 1.3645 \\
$\mathrm{~N}_{2}$ fraction & $6.82 \mathrm{E}-05$ & $3.31 \mathrm{E}-04$ & 0.001137 \\
$\mathrm{O}_{2}$ fraction & 0.9657 & 0.9642 & 0.9625 \\
Ar fraction & 0.0342 & 0.0355 & 0.0363 \\
Temperature (K) & 108.69 & 108.679 & 108.657 \\
Side-draw Product & & & \\
$\mathrm{N}_{2}$ fraction & 0.87674 & 0.87655 & 0.87988 \\
$\mathrm{O}_{2}$ fraction & 0.11575 & 0.11641 & 0.11347 \\
Ar fraction & 0.007503 & 0.007035 & 0.006642 \\
Temperature (K) & 97.113 & 97.12 & 97.808 \\
Reboiler duty (MW) & 28.5178 & 28.5168 & 28.3492 \\
\hline
\end{tabular}


Table 3: Specifications for optimization case study I

\begin{tabular}{ll}
\hline Chemical system & Feed specification \\
Binary system (Benzene-Toluene) & Flowrate: $100 \mathrm{kmol} / \mathrm{s}$ \\
Thermodynamics: Ideal & Composition $(\mathrm{mol} \%):(0.7,0.3)$ \\
& Temperature: $359.6 \mathrm{~K}$ \\
Column configuration & \\
Single feed & Additional constraints \\
Fixed Number of stages: 25 & Reflux ratio $\leq 1$ \\
Candidate stages for feed location: $\{8,9 \cdots 22\}$ & Top benzene purity $\geq 0.99$ \\
& \\
Operating conditions & Objective \\
Column Pressure: 1.12 bar & Maximize: Distillate $-50 \mathrm{r}$ \\
\hline
\end{tabular}

of output variables for the improved group method and the rigorous model match within three to four significant digits. Although the performance of the Edmister (1957) method is relatively good, it is less accurate particularly in the prediction of reboiler duty and temperatures. The use of better and realistic constraints guarantees that the improved group method will outperform the Edmister (1957) method for every distillation system and that too at no additional computational cost as the number of variables and equations remains unaffected. The strongest point in the favor of the improved group method is that we need not specify a priori whether the cascade of trays behaves as an absorber or a stripper whereas this specification is required for the Edmister (1957) method. Thus, the Edmister (1957) method may require several trials including knowledge of physical system and configuration while the improved group method provides a more accurate result with no additional insight needed.

\section{Optimization case studies}

The efficacy of the improved group method is also demonstrated using optimization case studies. The first case study involves finding the optimal feed location for a binary distillation column operating at constant pressure with fixed total number of trays. The specifications for this case study are described in Table 3. This problem is solved using both rigorous tray-by-tray model and the aggregate model. The representation for both these models is shown in Figure 3. The problem was originally solved by Viswanathan and Grossmann (1990) using the rigorous equilibrium stage model. Their model allows the feed to be inserted on all candidate feed location and uses binary variables and logic constraints to enforce that the feed is inserted on exactly one tray. Our aggregate model for this problem is based on using a fixed feed stage which is connected to a cascade of trays below and above it. The feed location is automatically changed by varying the 

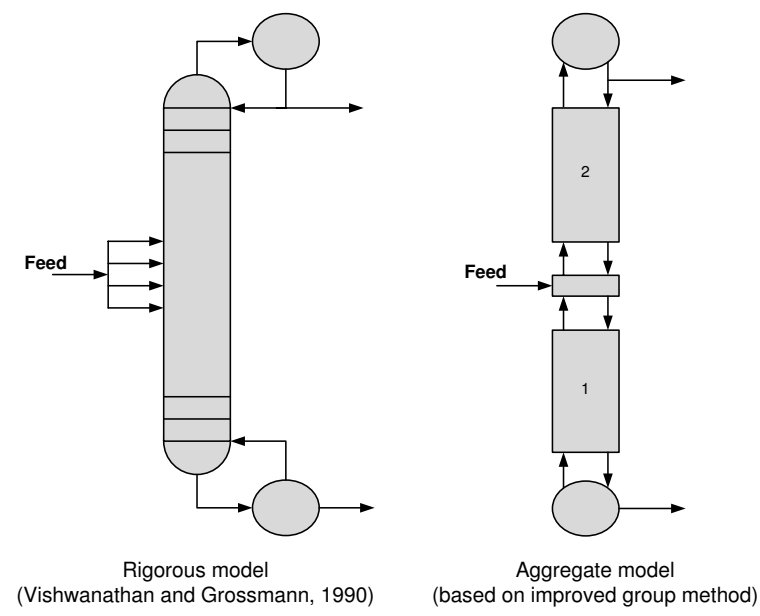

Figure 3: Rigorous and aggregate representations for the first optimization case study

number of trays in the two cascades. The total number of trays is fixed by enforcing that the sum of the number of trays in both the cascades is constant. Instead of binary variables, two integer variables are used to represent the number of stages in the cascades. The optimal solutions obtained using the rigorous and the aggregate model are shown in Table 4. The aggregate model can be solved in a sequence of steps. As a first step, the number of stages in the enriching and stripping cascades can be relaxed as continuous variables in order to solve a much simpler NLP problem. The solution of this NLP is shown in the third column of Table 4. The fractional values for enriching and stripping stages in the solution of this relaxed NLP can then be rounded off to the nearest integer values and the resulting NLP can be solved again with fixed integer values for the stages. We refer to this scheme as the rounding heuristic. The integer solution obtained by the rounding heuristic is shown in the fourth column of Table 4. Even if the rounding

Table 4: Results for optimization case study I

\begin{tabular}{lcccc}
\hline & $\begin{array}{c}\text { Rigorous Model } \\
\text { (Viswanathan and } \\
\text { Grossmann, 1990) }\end{array}$ & $\begin{array}{c}\text { Aggregate Model } \\
\text { (Relaxed) }\end{array}$ & $\begin{array}{c}\text { Aggregate Model } \\
\text { (Integer solution } \\
\text { by rounding) }\end{array}$ & $\begin{array}{c}\text { Aggregate Model } \\
\text { (best integer solution } \\
\text { by search) }\end{array}$ \\
\hline Objective & 20.34 & 21.96 & 21.821 & 21.821 \\
Reflux ratio & 0.985 & 0.975 & 0.978 & 0.978 \\
Distillate & 69.6 & 70.707 & 70.707 & 70.707 \\
Feed location & $15^{\text {th }}$ stage & - & $17^{\text {th }}$ stage & $17^{\text {th }}$ stage \\
Enriching stages & 13 & 14.9 & 15 & 15 \\
Stripping stages & 9 & 7.1 & 7 & 7 \\
Continuous variables & 235 & 90 & 88 & 88 \\
Discrete variables & 15 & - & - & 2 integer variables \\
& & & 80 & (but 15 candidates) \\
Total variables & 250 & 82 & 82 & 103 \\
Total constraints & 264 & 1 & 1 & 82 \\
Number of NLPs solved & 1 & 0.05 & 0.04 & 2 \\
CPU time (s) & 0.08 & & & 0.07 \\
\hline
\end{tabular}


Table 5: Specifications for optimization case study II

\begin{tabular}{ll}
\hline Chemical System & Operating conditions \\
Ternary system (Benzene-Toluene-o-xylene) & Reboiler Pressure: $1.25 \mathrm{bar}$ \\
Thermodynamics: Ideal & Condenser Pressure: $1.05 \mathrm{bar}$ \\
& Linear pressure profile \\
& \\
Column configuration & Additional constraints \\
Single feed & Distillate: $0.4 \mathrm{kmol} / \mathrm{s}$ \\
Maximum number of trays: 30 & Reflux ratio $\leq 15$ \\
& Bottom o-xylene purity $\geq 0.995$ \\
Feed specification & \\
Flowrate: $1 \mathrm{kmol} / \mathrm{s}$ & Objective \\
Composition $(\mathrm{mol} \%):(0.15,0.25,0.6)$ & Minimize: $5 \mathrm{r}+$ Stages \\
Temperature: $391.172 \mathrm{~K}$ & \\
\hline
\end{tabular}

heuristic is not used, all candidate integer solutions can still be searched using an outerapproximation or a branch-and-bound algorithm to determine the best integer solution. For this problem, the best integer solution turns out to be same as that given by the rounding heuristic. The number of variables and constraints in the aggregate model is almost one third of that in the rigorous model. In this case study, there is no noticeable difference in the computational time for the rigorous and the aggregate models. This is because the relaxed NLP for the rigorous model gives an integer solution and hence only one NLP is solved. While this can be treated as a heuristic and can be argued from an exergetic view point as negating the effect of mixing, it does not hold necessarily for all distillation systems. In spite of this, the NLPs for the aggregate model are smaller and solved marginally faster. We also see that the optimal feed location is missed by only two trays. The objective function of the aggregate model is overestimated by $7 \%$ due to the small differences in the reflux ratio and distillate flow rate.

In the second optimization case study, the total number of stages is also optimized along with the feed location. As compared to the case study I, this problem involves a ternary system and uses a pressure profile that varies linearly from the reboiler to the condenser. The specifications for this problem are given in Table 5 and the representations for the aggregate and rigorous models is shown in Figure 4. This problem was originally solved by Viswanathan and Grossmann (1993a) using the rigorous equilibrium stage model. Their model is based on fixing the feed at a particular stage and varying the location of the tray where the reflux and reboiled vapor enter by using corresponding binary variables. Trays above the entry point of the reflux and below the entry point of the reboiled vapor are automatically disabled. The strategy for our aggregate model is same as that described in case study I except that sum of the trays in the two cascade is not fixed but restricted by an upper bound. The computational results for this problem are shown in Table 6 . In this case, we observe that the number of variables and constraints in the aggregate model 


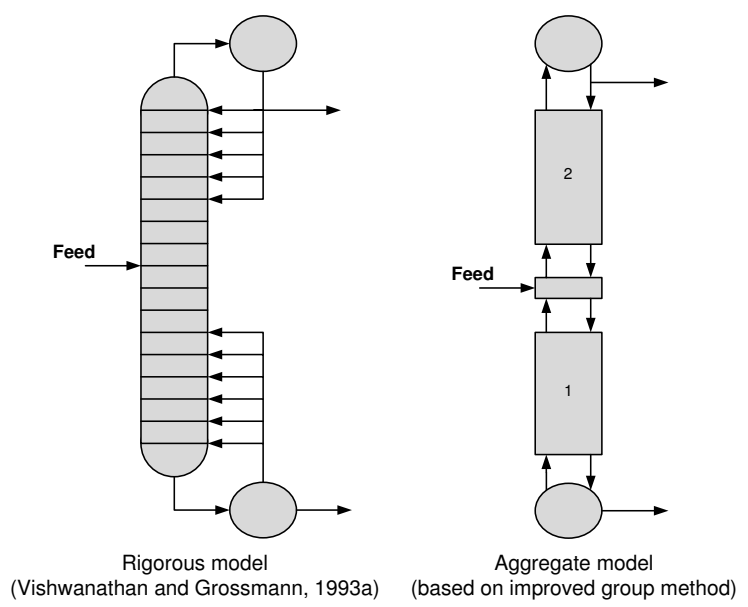

Figure 4: Rigorous and aggregate representations for the second optimization case study

is almost one fourth of that in the rigorous model. It is also significantly faster consuming less than a second as compared to sixteen seconds for the rigorous model. The aggregate model predicts one additional tray and the feed location is also missed by only one tray. Just like the first case study, the best integer solution found by rigorous search is same as that given by the rounding heuristic. The optimal objective function of aggregate model in this case is overestimated by around $8.5 \%$. It is interesting to note that if the rigorous model is solved with a fixed structural configuration corresponding to that of the optimal aggregate solution, the objective increases by only $0.28 \%$, reflux is reduced by $7.81 \%$ while the computational time is $0.4 \mathrm{CPU}$ seconds since only one rigorous NLP is solved. Thus, it may also be worthwhile to use the optimal solution of the aggregate model to fix the structural configuration and solve the rigorous model as a much simpler NLP problem.

Note that the previous two case studies used ideal thermodynamics for vapor liquid equi-

Table 6: Results for optimization case study II

\begin{tabular}{lcccc}
\hline & $\begin{array}{c}\text { Rigorous model } \\
\text { (Viswanathan and } \\
\text { Grossmann, 1993a) }\end{array}$ & $\begin{array}{c}\text { Aggregate model } \\
\text { (Relaxed) }\end{array}$ & $\begin{array}{c}\text { Aggregate model } \\
\text { (Integer solution } \\
\text { by rounding) }\end{array}$ & $\begin{array}{c}\text { Aggregate model } \\
\text { (Best integer solution } \\
\text { by search) }\end{array}$ \\
\hline Objective & 28.76 & 31.2 & 31.2266 & 31.2266 \\
Reflux ratio & 2.35 & 2.72 & 2.645 & 2.645 \\
Number of stages & 17 & 17.58 & 18 & 18 \\
Feed location & $9^{\text {th }}$ stage & - & $10^{\text {th }}$ stage & $10^{\text {th }}$ stage \\
Enriching stages & 7 & 7.73 & 8 & 8 \\
Stripping stages & 9 & 8.85 & 9 & 9 \\
Continuous variables & 400 & 109 & 107 & 107 \\
Discrete variables & 23 & - & - & 2 integer variables \\
Total variables & 423 & 109 & 107 & (but 23 candidates) \\
Total constraints & 474 & 99 & 99 & 99 \\
Number of NLPs solved & 150 & 1 & 1 & 6 \\
CPU time (s) & 15.92 & 0.04 & 0.02 & 0.11 \\
\hline
\end{tabular}


Table 7: Specifications for optimization case study III

\begin{tabular}{ll}
\hline Chemical System & Operating conditions \\
Ternary system $(n$-hexane- $n$-heptane- $n$-nonane) & Feed pressure: $1.7301 \mathrm{bar}$ \\
Thermodynamics: RKS cubic EOS & Reboiler pressure: $1.7404 \mathrm{bar}$ \\
& Condenser pressure: $1.3785 \mathrm{bar}$ \\
Column configuration & Linear pressure profile \\
Two feeds & Additional constraints \\
Maximum number of trays: 35 & Reflux ratio $\leq 5$ \\
& Heptane in top $\leq 1 \%$ of feed \\
Feed 1 specification & Hexane in bot $\leq 1 \%$ of feed \\
Flowrate: $50 \mathrm{kmol} / \mathrm{s}$ & Objective \\
Composition $(\mathrm{mol} \%):(0.3,0.1,0.6)$ & Minimize: $3.64 \mathrm{E}-3 \mathrm{QReb}+$ Stages +2 \\
Temperature: bubble point & \\
& \\
Feed 2 specification & \\
Flowrate: $50 \mathrm{kmol} / \mathrm{s}$ & \\
Composition $(\mathrm{mol} \%):(0.4,0.3,0.3)$ & \\
Temperature: bubble point &
\end{tabular}

librium (VLE). In order to demonstrate that the modified group method works fairly well even for nonideal systems, we present a third case study that uses non-ideal thermodynamics in form of Soave-Redlich-Kwong (SRK) cubic equation of state. This problem involves two feeds instead of a single feed as discussed in the previous two case studies. The objective of this problem is same as that of case study II, i.e. to find the optimum total number of stages and feed locations. The specifications for this problem are given in Table 7 while the representation of the rigorous and aggregate models is shown in Figure 5. This problem was originally solved by Viswanathan and Grossmann (1993b) using the rigorous equilibrium stage model. This model is similar to the rigorous model in the previous case study except that the location of the reboiled vapor is fixed and only the location of the reflux is allowed to vary. The structure of the aggregate model, on the other hand is slightly different from the previous two case studies because of the presence of two feeds which requires an additional cascade of trays. Additional mixers and splitters are introduced to account for the possibility that any of the two feeds could be placed at the top or the bottom of the intermediate cascade. Binary variables are used for splitter outlets along with logic constraints to enforce that exactly one feed is introduced on each of the feed stages. Since group methods require a minimum of two trays in a cascade, the aggregate model cannot handle cases where the feed locations differ by less than two trays. The computational results for this problem are shown in Table 8. The number of variables and constraints in the aggregate model is almost one fourth of that in the rigorous model. In terms of computational speed, the aggregate model is a clear winner consuming just 0.68 seconds as compared to 20 mins for the rigorous model. The total number of stages is the same while the feed tray location is missed by only two trays for the first feed and one tray for the second feed. The deviation in the condenser and reboiler 

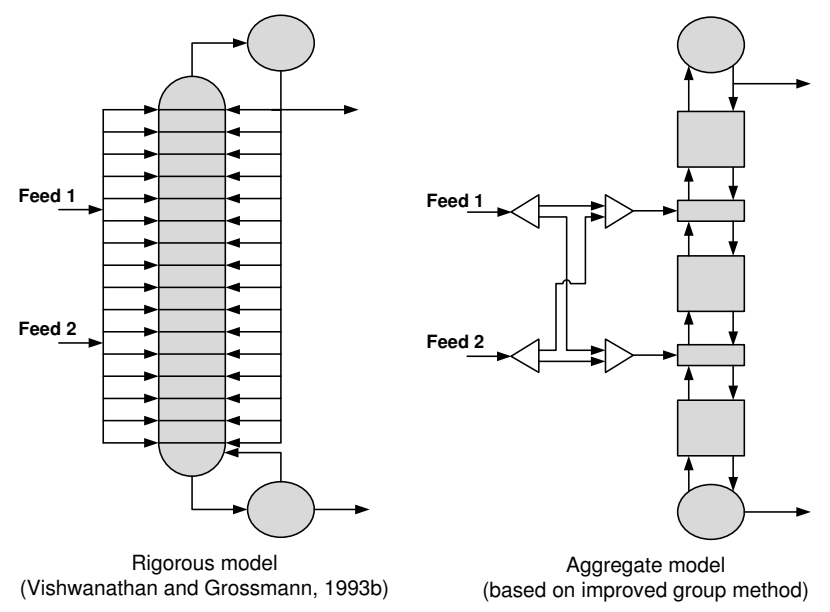

Figure 5: Rigorous and aggregate representations for the third optimization case study

loads is around $10 \%$. As was reported for the second optimization case study, if the rigorous model is solved as an NLP using a fixed structural configuration corresponding to the optimal aggregate solution, it is found that the objective function increases by only $1.3 \%$. Furthermore, the comparison between the rigorous and the aggregate model can be made more fair by introducing additional logic constraints in the rigorous model to enforce that the feed locations differ by at least two trays. The optimal solution of this restricted rigorous model is shown in the last column of Table 8. As can be seen, when the same restriction on the feed location is used for both models, it is observed that only one feed location is missed by a single tray and the difference in the performance of the aggregate and rigorous models reduces further. Finally, if the rigorous model in these optimization case studies are solved as simulation problems by fixing degrees of freedom (reflux ratio,

Table 8: Results for optimization case study III

\begin{tabular}{|c|c|c|c|c|c|}
\hline & $\begin{array}{l}\text { Rigorous model } \\
\text { (Viswanathan and } \\
\text { Grossmann, 1993b) }\end{array}$ & $\begin{array}{l}\text { Aggregate model } \\
\text { (Relaxed) }\end{array}$ & $\begin{array}{c}\text { Aggregate model } \\
\text { (Integer solution } \\
\text { by rounding) }\end{array}$ & $\begin{array}{c}\text { Aggregate model } \\
\text { (Best integer solution } \\
\text { by search) }\end{array}$ & $\begin{array}{l}\text { Rigorous model } \\
\text { (Restriction on } \\
\text { feed location) }\end{array}$ \\
\hline Objective & 32.557 & 33.936 & 34.0657 & 33.9838 & 32.777 \\
\hline Number of stages & 20 & 19.84 & 19 & 20 & 20 \\
\hline Reflux ratio & 2.641 & 3.061 & 3.326 & 3.03 & 2.701 \\
\hline Condenser duty (MW) & 3.661 & 4.084 & 4.35 & 4.053 & 3.721 \\
\hline Reboiler duty (MW) & 3.999 & 4.422 & 4.688 & 4.391 & 4.059 \\
\hline Feed 1 location & $10^{\text {th }}$ stage & - & $8^{\text {th }}$ stage & $8^{\text {th }}$ stage & $9^{\text {th }}$ stage \\
\hline Feed 2 location & $11^{\text {th }}$ stage & - & $11^{\text {th }}$ stage & $12^{\text {th }}$ stage & $12^{\text {th }}$ stage \\
\hline Continuous variables & 1226 & 318 & 315 & 315 & 1226 \\
\hline Discrete variables & 99 & - & - & $\begin{array}{l}3 \text { integer variables } \\
\text { (but } 72 \text { candidates) }\end{array}$ & $\begin{array}{l}3 \text { integer variables } \\
4 \text { binary variables }\end{array}$ \\
\hline Total variables & 1325 & 318 & 315 & 387 & 1325 \\
\hline Total constraints & 1494 & 270 & 270 & 270 & 1659 \\
\hline Number of NLPs solved & 1522 & 1 & 1 & 10 & 1274 \\
\hline CPU time $(\mathrm{s})$ & 1246 & 0.16 & 0.03 & 0.68 & 1045 \\
\hline
\end{tabular}


Table 9: Comparison of optimal solution of aggregate model and corresponding simulation using rigorous model

\begin{tabular}{lcccc}
\hline & \multicolumn{2}{c}{ Optimization case study II } & \multicolumn{2}{c}{ Optimization case study III } \\
& Aggregate solution & Rigorous simulation & Aggregate solution & Rigorous simulation \\
\hline Top temperature (K) & 370.473 & 370.425 & 352.324 & 352.294 \\
Bottom temperature (K) & 425.297 & 425.412 & 421.058 & 421.119 \\
Condenser duty (MW) & 4.8813 & 4.8752 & 4.0534 & 4.0517 \\
Reboiler duty (MW) & 5.2194 & 5.2144 & 4.3912 & 4.3906 \\
\hline
\end{tabular}

top flowrate) and structural configuration corresponding to the optimal solution of the aggregate model, then important output variables like heat loads and temperatures do not differ significantly. This is demonstrated in Table 9 and further emphasizes that the aggregate model can be used with sufficient reliability for simulation case studies.

\section{Conclusions}

This work has addressed the use of aggregate models for complex distillation columns with the aim of simplifying or reducing computational effort without significant loss of accuracy and reliability of the desired objective. Group methods are identified as one of the very few aggregate methods that can not only provide reasonably accurate predictions, but also handle mixed-integer optimization problems like determining optimum number of trays and feed locations. With the aid of systematic degree of freedom analysis, the previously proposed approximations used in group methods were analyzed and only the ones fundamental to the method were retained whereas certain approximations related to absorption or stripping are replaced by more realistic constraints based on the principles of distillation. As a result, the improved group method turns into a general purpose model for a cascade of trays in counter-current gas liquid operations. The most important advantage, as compared to previous work is that the behavior of the cascade of trays as an absorber or a stripper need not be specified a priori.

The capability of the aggregate models developed using the improved group method was demonstrated using simulation and optimization capabilities. These aggregate models have fewer variables and constraints thereby resulting in smaller problems that are much easier to solve. In spite of their approximation nature, the output variables of the aggregate model are in close agreement with that of the rigorous model. For the optimization case studies, it was observed that the optimal number of trays and feed locations are missed by only one or two trays. For such problems, the aggregate model can be applied 
in a sequence of steps starting from a relaxed NLP problem to a rigorous search of all integer solutions. This stepwise application leads to a robust and reliable solution procedure. A rounding heuristic was also proposed, which is based on solving the relaxed NLP and then rounding off the solution to the nearest integer. The rounding heuristic often generates near-optimal solutions with a significant reduction in computational time. Since the case studies involved different kinds of binary and ternary systems, ideal and nonideal thermodynamics, and a wide variety of structural and operating conditions, we expect that the aggregate models will work well on many related problems. 


\section{References}

AspenTech, Aspen Plus User Manual, Version 2006.5, Aspen Tech (2006).

Bagajewicz, M. J. and V. Manousiouthakis, "Mass/Heat exchange Network representation of Distillation Networks," AIChE J., 38, 1769-1800 (1992).

Barttfeld, M., P. A. Aguirre, and I. E. Grossmann, "Alternative representations and formulations for the economic optimization of multicomponent distillation columns," Comput. Chem. Eng., 27, 363-383 (2003).

Caballero, J. A. and I. E. Grossmann, "Aggregated Models for Integrated Distillation Systems," AIChE J., 38, 2330-2344 (1999).

Edmister, W. C., "Design of Hydrocarbon Absorption and Stripping," Ind. Eng. Chem., 35, 837-839 (1943).

Edmister, W. C., "Absorption and Stripping Factor Functions for distillation calculation by manual and digital computer methods," AIChE J., 3, 165-171 (1957).

Fenske, M. R., "Fractionation of straight-run Pennsylvania gasoline," Ind. Eng. Chem. Res., 24, 482-485 (1932).

Gilliland, E. R., "Multicomponent rectification: estimation of the number of theoretical plates as a function of the reflux ratio," Ind. Eng. Chem. Res., 32, 1220-1223 (1940).

Henley, E. J. and J. D. Seader, Equilibrium-Stage Separation Operations in Chemical Engineering. Wiley (1981).

Kremser, A., "Theoretical analysis of absorption process," Nat. Petroleum News, 22, 43-49 (1930).

Lang, Y.-D. and L. T. Biegler, "Distributed Stream Method for Tray Optimization," AIChE J., 48, 582-595 (2002).

Papalexandri, K. P. and E. N. Pistikopoulos, "Generalized Modular representation Framework for Process Synthesis," AIChE J., 42, 1010-1032 (1996).

Underwood, A. J. V., "Fractional Distillation of Multicomponent Mixtures," Chem. Eng. Progress, 44, 603-614 (1948). 
Viswanathan, J. and I. E. Grossmann, "A Combined Penalty Function and OuterApproximation Method for MINLP Optimization," Comput. Chem. Eng., 14, 769-782 (1990).

Viswanathan, J. and I. E. Grossmann, "An Alternate MINLP Model for Finding the Number of Trays Required for a Specified Separation Objective," Comput. Chem. Eng., 17, 949-955 (1993a).

Viswanathan, J. and I. E. Grossmann, "Optimal Feed Locations and Number of Trays for Distillation Columns with Multiple Feeds," Ind. Eng. Chem. Res., 32, 2942-2949 (1993b).

Yeomans, H. and I. E. Grossmann, "Disjunctive Programming Models for the Optimal Design of Distillation Columns and Separation Sequences," Ind. Eng. Chem. Res., 39, 1637-1648 (2000). 